\title{
Pratiques éducatives pour accroitre la participation sociale des adolescents et des jeunes adultes ayant un trouble du spectre de l'autisme
}

\section{Educational Practices to Increase Social Participation of Adolescents and Young Adults with Autism Spectrum Disorder}

\author{
Daphné Rouillard-Rivard, Francine Julien-Gauthier, Marie-Hélène Poulin et \\ Sarah Martin-Roy
}

Volume 47, numéro 1, 2018

URI : https://id.erudit.org/iderudit/1046771ar

DOI : https://doi.org/10.7202/1046771ar

Aller au sommaire du numéro

\section{Éditeur(s)}

Revue de Psychoéducation

ISSN

1713-1782 (imprimé)

2371-6053 (numérique)

Découvrir la revue

Citer cet article

Rouillard-Rivard, D., Julien-Gauthier, F., Poulin, M.-H. \& Martin-Roy, S. (2018). Pratiques éducatives pour accroitre la participation sociale des adolescents et des jeunes adultes ayant un trouble du spectre de l'autisme. Revue de psychoéducation, 47(1), 23-52. https://doi.org/10.7202/1046771ar

\section{Résumé de l'article}

Bien que la participation sociale soit un des objectifs des services éducatifs offerts lors de la scolarisation, peu de recherches se sont penchées sur cet enjeu, et ce, plus particulièrement pour les adolescents et les jeunes adultes ayant un trouble du spectre de l'autisme (TSA). Cette recension des écrits porte sur la participation sociale de ces adolescents et jeunes adultes (14 à 21 ans) dans une perspective de résilience. Elle a pour but d'identifier des facteurs de risque susceptibles d'entraver leur participation sociale et des facteurs de protection qui peuvent la favoriser. Pour la réalisation de l'étude, 31 articles scientifiques et un chapitre de livre ont été recensés. À la lumière des résultats obtenus, la période développementale de l'adolescence représente une fenêtre d'occasions pour le développement de la participation sociale, car les adolescents et les jeunes adultes côtoient quotidiennement leurs pairs dans le milieu scolaire. À l'adolescence, le jeune se distancie de la cellule familiale pour construire son identité personnelle en établissant des liens avec ses pairs; dans cette perspective les habiletés sociales sont étroitement liées à la participation sociale. Les résultats obtenus confirment l'importance de la participation sociale pour les jeunes ayant un TSA, de même que la nécessité de soutenir cette participation sociale par l'apprentissage d'habiletés sociales et la multiplication des occasions de les mettre en pratique à l'école et dans leur collectivité. Des stratégies d'intervention à mettre en place pour accroitre la participation sociale de ces jeunes sont proposées.
Tous droits réservés @ La Corporation de la Revue Canadienne de Psycho-Éducation, 2018
Ce document est protégé par la loi sur le droit d'auteur. L’utilisation des services d’Érudit (y compris la reproduction) est assujettie à sa politique d'utilisation que vous pouvez consulter en ligne. 


\title{
Pratiques éducatives pour accroitre la participation sociale des adolescents et des jeunes adultes ayant un trouble du spectre de l'autisme
}

\author{
Educational Practices to Increase Social Participation of \\ Adolescents and Young Adults with Autism Spectrum Disorder
}

\section{Rouillard- Rivard $^{1}$ \\ F. Julien-Gauthier ${ }^{1}$ M.-H. Poulin ${ }^{2}$ S. Martin-Roy ${ }^{1}$}

\author{
Université Laval \\ 2 Université du Québec en \\ Abitibi-Témiscamingue
}

\section{Correspondance :}

Daphné Rouillard-Rivard 2320 , rue des Bibliothèques Université Laval Québec (Québec) G1V 0A6

Téléphone : 418-208-1462 daphne.rouillard-rivard.1@ ulaval.ca

\section{Résumé}

Bien que la participation sociale soit un des objectifs des services éducatifs offerts lors de la scolarisation, peu de recherches se sont penchées sur cet enjeu, et ce, plus particulièrement pour les adolescents et les jeunes adultes ayant un trouble du spectre de l'autisme (TSA). Cette recension des écrits porte sur la participation sociale de ces adolescents et jeunes adultes (14 à 21 ans) dans une perspective de résilience. Elle a pour but d'identifier des facteurs de risque susceptibles d'entraver leur participation sociale et des facteurs de protection qui peuvent la favoriser. Pour la réalisation de l'étude, 31 articles scientifiques et un chapitre de livre ont été recensés. À la lumière des résultats obtenus, la période développementale de l'adolescence représente une fenêtre d'occasions pour le développement de la participation sociale, car les adolescents et les jeunes adultes côtoient quotidiennement leurs pairs dans le milieu scolaire. À l'adolescence, le jeune se distancie de la cellule familiale pour construire son identité personnelle en établissant des liens avec ses pairs; dans cette perspective les habiletés sociales sont étroitement liées à la participation sociale. Les résultats obtenus confirment l'importance de la participation sociale pour les jeunes ayant un TSA, de même que la nécessité de soutenir cette participation sociale par l'apprentissage d'habiletés sociales et la multiplication des occasions de les mettre en pratique à l'école et dans leur collectivité. Des stratégies d'intervention à mettre en place pour accroitre la participation sociale de ces jeunes sont proposées.

Mots-clés : participation sociale; trouble du spectre de l'autisme; facteurs de risque et de protection; pratiques éducatives; résilience.

\section{Abstract}

Although social participation is one of the objectives of educational services provided during schooling, few researches have been conducted on this issue, especially for adolescents and young adults with autism spectrum 
disorders (ASD). This literature review focuses on the social participation of these adolescents and young adults (aged 14 to 21) from a perspective of resilience. This research aims to identify risk factors that may hinder their social participation and the protective factors that may favor it. To conduct this study, 31 scientific articles and one book chapter were identified. Our results demonstrate that adolescence, as a developmental period, represents a window of opportunity for the development of social participation, as adolescents and young adults frequent their peers daily in the school environment. As a teenager, the youngster distances himself from the family unit to build his own identity by establishing links with his peers. From this perspective, social skills are closely linked to social participation. Our results confirm the importance of social participation for youth with ASD, as well as the need to support their social participation through the learning of social skills and the multiplication of opportunities to put them into practice at school and in the community. Intervention strategies are proposed to increase the social participation of these young people.

\section{Keywords: social participation; autism spectrum disorder; risk and protective fac- tors; educational practices; resilience.}

\section{Introduction}

Au cours des dernières années, la fréquence du trouble du spectre de l'autisme est d'environ $1 \%$ de la population, avec des estimations comparables entre les échantillons d'enfants et d'adultes (American Psychiatric Association, 2015). Au Québec, il s'agit de la catégorie d'élèves handicapés ou en difficulté d'adaptation ou d'apprentissage (ÉHDAA) ayant la plus grande prévalence dans le secteur de l'instruction publique (Noiseux, 2008). Les personnes ayant un TSA présentent des déficits persistants de la communication et des interactions sociales, ainsi qu'un caractère restreint et répétitif des comportements, des intérêts et des activités (American Psychiatric Association, 2015). Les symptômes se présentent selon un continuum de sévérité d'où l'appellation de « spectre ». Selon le DSM 5 , le niveau de soutien requis pour répondre à leurs besoins varie de léger à très important. Les défis rencontrés par les jeunes ayant un TSA lors de la transition vers la vie active sont documentés dans plusieurs études (Hendricks et Wehman, 2009; Schall, CortijoDoval, Sherron Targett et Wehman, 2006; Shattuck, Orsmond, Wagner et Cooper, 2011). Les principaux défis auxquels ils sont confrontés lors de cette transition comprennent le besoin de développer leur autonomie, de s'investir dans un travail, d'entreprendre des études postsecondaires, de s'engager dans leur communauté et de surmonter des situations d'adversité, notamment l'isolement social (Liptak, Kennedy et Dosa, 2011). À la fin de la scolarisation, les élèves quittent le milieu scolaire afin d'intégrer de nouveaux milieux de vie. Cette transition les amène à vivre des changements critiques relativement au maintien et au développement de leur réseau social. En effet, il s'avère difficile pour les jeunes ayant un TSA de maintenir, à l'extérieur du milieu scolaire, les relations interpersonnelles auparavant développées avec leurs pairs à l'école (Orsmond, Shattuck, Cooper, Sterzing et Anderson, 2013). De plus, Myers, Davis, Stobbe et Bjornson (2015) ont observé une diminution significative de la participation communautaire (participation à des activités à l'extérieur de la classe ou de l'école) de ces jeunes lors du passage de l'adolescence à l'âge adulte (63 à $46 \%$ ). 
L'un des objectifs principaux des services offerts en milieu scolaire ou de réadaptation est de soutenir et de favoriser la participation sociale des élèves (Carter, Harvey, Taylor et Gotham, 2013; Gouvernement du Québec, 2006; 2016; 2017; Proulx, 2008; Test, Smith et Carter, 2014; Wehman et al., 2014). Les intervenants en milieu scolaire doivent intervenir auprès de l'élève ayant des besoins particuliers afin de faciliter la transition vers la vie active et favoriser le développement des compétences fonctionnelles nécessaires pour évoluer au sein d'un milieu communautaire, de travail ou de formation à la fin de la scolarisation (Carter, Harvey, Taylor et Gotham, 2013). Ainsi, la participation sociale est un objectif majeur des services éducatifs et sociaux offerts aux jeunes vivant avec une incapacité, et celleci nécessite un arrimage entre le jeune, sa famille, l'entourage et les milieux de vie (Tétreault, 2015). Toutefois, malgré l'importance de la participation sociale de ces jeunes pour l'accès à une vie active après la scolarisation (Julien-Gauthier, Héroux et Jourdan-Ionescu, 2012), peu de recherches ont permis d'identifier des stratégies efficaces pour la favoriser (Hendricks et Wehman, 2009). Documenter la participation sociale de ces jeunes est donc primordial, car elle constitue un aspect central de leur vie (Orsmond et al., 2013) et un défi de taille pour leur avenir.

Cette recension des écrits scientifiques a pour but de mieux connaître le contexte influençant la participation sociale des adolescents et jeunes adultes (1421 ans) ayant un TSA, donc de répondre aux questions suivantes : 1) Quels sont les facteurs de risque et les facteurs de protection à la participation sociale des adolescents et jeunes adultes ayant un TSA? et 2) Quelles stratégies d'intervention permettent de faciliter la participation sociale de ces jeunes dans les divers milieux dans lesquels ils évoluent?

\section{Contexte théorique}

Tel que mentionné plus tôt, la participation sociale est un des principaux buts de l'aide ou des services offerts aux personnes ayant un trouble du spectre de l'autisme (Gouvernement du Québec, 2016; 2017). Toutefois, leur participation sociale est une notion qui demeure abstraite, peu documentée et qui requiert une réflexion approfondie (Fédération québécoise des centres de réadaptation en déficience intellectuelle et en troubles envahissant du développement [FQCRDITED], 2013). La définition de la participation sociale privilégiée dans cet article s'inspire des travaux de Hall $(2009,2010)$, de Novak, Stancliffe, McCarron et McCallion (2013), de Lysaght, Cobigo et Hamilton (2012) et de résultats d'études au sujet de la participation sociale des élèves ayant des incapacités, dans une perspective de résilience (Martin-Roy et Julien-Gauthier, 2017; Rouillard-Rivard, 2016; StGeorges, 2017). La participation sociale fait référence : 1) à leur participation à des activités significatives dans différents contextes de vie selon leur âge et leur culture; 2) à l'établissement et au maintien de relations réciproques avec les membres de leur communauté; et 3) à la présence d'un sentiment d'appartenance à des groupes ou à des réseaux sociaux (Julien-Gauthier, Martin-Roy, Moreau, Ruel et RouillardRivard, 2016). 
Le cadre théorique de la résilience est utilisé dans cette étude en raison des liens étroits entre la participation sociale et la résilience (Rouillard-Rivard, Julien-Gauthier et Martin-Roy, 2016). Lors de la transition vers la vie adulte, les adolescents et les jeunes adultes ayant un TSA sont confrontés à ses situations d'adversité qui entravent leur participation sociale actuelle et future (RouillardRivard, 2016). Pour les aider à surmonter les difficultés rencontrées, une intervention écosystémique axée sur la résilience est à privilégier (Jourdan-lonescu, 2001; 2017). II convient d'abord d'identifier avec l'élève et son entourage la présence de facteurs de risque et de protection individuels, familiaux ou environnementaux. Des stratégies d'intervention sont ensuite élaborées afin de diminuer l'impact des facteurs de risque, de développer et de consolider les facteurs de protection. Les interventions de résilience assistée sont co-construites en partenariat avec l'élève et son entourage et visent la mise en place d'actions individualisées, qui s'appuient sur des facteurs de protection trans-adversités (Jourdan-lonescu, 2017). II peut s'agir de la mise en relation de la personne avec un ou des tuteurs de résilience (Ionescu, 2015), de l'utilisation de l'humour pour relativiser une situation (JourdanIonescu et Julien-Gauthier, 2011) ou de la référence à des structures sociales facilitantes, tels les programmes de développement des habiletés sociales ou les organismes de loisirs (Julien-Gauthier et al., 2014). Ces stratégies se caractérisent par un véritable accompagnement de l'élève qui, en facilitant l'actualisation de ses compétences et leur utilisation pour faire face à l'adversité, façonne et renforce sa résilience naturelle ${ }^{1}$ (Ionescu, 2011).

Dans cette perspective, la participation sociale des adolescents et des jeunes adultes ayant un TSA revêt une importance capitale, puisqu'elle favorise l'interaction avec l'entourage et le développement de liens sociaux (Julien-Gauthier, Moreau, Martin-Roy, Ruel et Rouillard-Rivard, 2015). Toutefois, la nature de cette participation sociale, les défis qui y sont associés et les conditions qui la favorisent sont peu connus. Cette recension des écrits scientifiques permet de mettre en évidence des facteurs de risque ${ }^{2}$, qui peuvent entraver la participation sociale des jeunes ayant un TSA et des facteurs de protection, susceptibles de faciliter son développement et sa consolidation, ainsi que des stratégies d'intervention pour assurer la réussite de la transition vers une vie active, à la fin de la scolarisation. Les stratégies qui favorisent la participation sociale de ces jeunes leur permettent, dans l'interaction avec l'entourage, de surmonter les situations d'adversité, de renforcer leur résilience naturelle et d'envisager l'avenir de façon confiante et positive (JulienGauthier, Ruel, Moreau et Martin-Roy, 2016).

1. La résilience naturelle intervient sans aucune aide de la part de professionnels (lonescu, 2011). Elle se développe dans la confrontation au risque (Jourdan-Ionescu, 2017) par l'interaction entre la personne, sa famille et son environnement social, physique ou culturel.

2. Un facteur de risque est un élément qui appartient à la personne ou qui provient de l'environnement, susceptible de porter atteinte à son intégrité ou à son développement (Fougeyrollas, 2010). Quant au facteur de protection, il peut être défini en tant qu'élément qui appartient à la personne ou qui provient de son environnement, susceptible de préserver son intégrité physique et de favoriser son développement ou son épanouissement dans sa collectivité (Julien-Gauthier et al., 2016). 


\section{Méthode}

Cette recension des écrits scientifiques " narrative " vise à connaitre l'état d'avancement de la recherche (Tétreault, 2014) au sujet de la participation sociale des adolescents et des jeunes adultes (14-21 ans) ayant un TSA. Les résultats se limitent aux études scientifiques qui ont été publiées.

Six critères d'inclusion ont été utilisés : (a) les articles ont été publiés entre 2005 et 2015; (b) les participants aux études sont majoritairement (plus de $50 \%$ ) des adolescents ou des jeunes adultes âgés de 14 à 21 ans ayant reçu un diagnostic de TSA selon le DSM-5 ou de trouble envahissant du développement selon le DSM-IV-TR; (c) les articles sont des recensions d'écrits scientifiques ou des études empiriques qualitatives ou quantitatives publiées dans des revues scientifiques avec comité de pairs; (d) les auteurs tentent de définir ou de décrire la participation sociale des adolescents ou des jeunes adultes ayant un TSA ou abordent un thème central au développement de la participation sociale (ex. : les compétences sociales, la communication, le développement vocationnel, les activités de la vie quotidienne, la vie communautaire), (e) les articles sont accessibles à l'aide des ressources électroniques de la bibliothèque de l'Université Laval et (f) la langue de publication est l'anglais ou le français.

Afin de déterminer les revues scientifiques susceptibles de publier des articles concernant la participation sociale des adolescents et des jeunes adultes ayant un TSA, une recherche dans Ariane $2.0^{3}$ a été réalisée. Le mot-clé autism* accompagné de la spécification Titre de périodique a permis d'identifier les revues scientifiques pertinentes. Les derniers numéros (2013-2015) des revues suivantes ont été consultés: Intellectual and Developmental Disabilities, Autism, Journal of Autism and Developemental Disorders, Focus on Autism and Other Developmental Disabilities et Autism Research. La recherche a permis de repérer 33 articles à partir des titres. La lecture des résumés de ces articles et, au besoin, une vérification de la méthode utilisée a permis d'exclure 18 articles ne correspondant pas aux critères d'inclusion. Au total, 15 articles scientifiques provenant des revues scientifiques pertinentes sont retenus.

Par la suite, les bases de données suivantes ont été consultées (années 2005 à 2015) : PsycINFO, ERIC et Medline. La recherche a été réalisée à partir des mots-clés " autism* " OR « ASD » AND " social participation » en combinaison avec « adolescen* » et " young adult » ou « young adulthood ». À partir des titres, la consultation a permis de répertorier 34 articles dont plusieurs recensés dans les revues scientifiques consultées se retrouvaient également dans les résultats de recherche des bases de données interrogées. Certains articles issus de la recherche dans les bases de données se retrouvaient également dans plusieurs d'entre elles ainsi que conjointement dans les résultats issus de la combinaison avec " adolescen* " et " young adult* ». Une première lecture des résumés de chacun des articles électroniques recensés a aussi permis d'exclure certains articles qui ne répondaient pas aux critères d'inclusion. Au total 10 articles ont été retenus.

3. Ariane 2.0 est le catalogue en ligne de la bibliothèque de l'Université Laval. 
Enfin, la lecture des 25 articles recensés dans les revues scientifiques pertinentes $(N=15)$ et dans les bases de données $(N=10)$ a permis d'identifier, dans leurs listes de références respectives, des articles et des ouvrages à consulter. De ce fait, six autres articles et un chapitre de livre ont été ajoutés à la recension des écrits scientifiques. Un total de 31 articles scientifiques et un chapitre de livre traitant de la participation sociale des adolescents et des jeunes adultes ayant un TSA ont été recensés. Certains articles ont été retenus malgré le fait qu'ils ne correspondent pas complètement aux critères d'inclusion en raison de leur pertinence pour l'avancement des connaissances sur la participation sociale des adolescents et jeunes adultes ayant un TSA. Ces articles sont identifiés par un astérisque devant le nom des auteurs dans le tableau 1.

\section{Discussion}

\section{Facteurs de risque et de protection}

Dans une perspective de résilience, les résultats de cette recension des écrits ont permis d'identifier des facteurs de risque et des facteurs de protection pour la participation sociale des adolescents et des jeunes adultes ayant un TSA.

FACTEURS DE RISQUE
$\begin{aligned} & \text { Individuels } \\ & \text { Les caractéristiques du TSA }\end{aligned}$
$\begin{aligned} & \text { Lisolement social } \\ & \text { La difficultéa participer à la planification } \\ & \text { de la transition vers la vie active }\end{aligned}$
$\begin{aligned} & \text { Familiaux } \\ & \text { La difficulté à identifier les ressources pertinentes } \\ & \text { pour lintégration communautaire }\end{aligned}$
$\begin{aligned} & \text { Environnementaux } \\ & \text { La méconnaissance par les intervenants des } \\ & \text { interventions probantes pour soutenir les élèves } \\ & \text { qui ont un TSA }\end{aligned}$
$\begin{aligned} & \text { Les croyances erronées des intervenants au sujet } \\ & \text { de remploi }\end{aligned}$
$\begin{aligned} & \text { La méconnaissance et lincompréhension des pairs } \\ & \text { des déficits sociaux }\end{aligned}$

Figure 1 : Facteurs de risque et de protection pour favoriser la participation sociale des adolescents et des jeunes adultes ayant un TSA, à partir des résultats de la recension des écrits

\section{Pratiques éducatives pour favoriser la participation sociale}

Dans cette section sont présentées des stratégies d'intervention qui permettent de réduire les facteurs de risque ou leur portée ainsi que de développer ou de consolider des facteurs de protection pour faciliter la participation sociale de l'adolescent ou du jeune adulte ayant un TSA, et conséquemment, de renforcer sa résilience naturelle. Celle-ci est un type de résilience qui se construit durant l'ontogénèse, sans l'aide de cliniciens ou de professionnels de la santé (lonescu, 2011). Elle n'est pas innée, mais se développe lors de la confrontation au risque, 


\section{Résultats}

\section{Tableau 1. Recension des écrits sur la participation sociale (PS) des adolescents et jeunes adultes ayant un TSA}

\begin{tabular}{|c|c|c|c|c|}
\hline $\begin{array}{l}\text { Auteurs et } \\
\text { année de } \\
\text { publication }\end{array}$ & Objectifs & Méthodes & Participants & Principaux résultats \\
\hline $\begin{array}{l}\text { Anderson, } \\
\text { Shattuck, } \\
\text { Cooper, Roux } \\
\text { et Wagner } \\
(2014)\end{array}$ & $\begin{array}{l}\text { Étudier la } \\
\text { prévalence et } \\
\text { les corrélations } \\
\text { de trois modes } \\
\text { de vie de jeunes } \\
\text { adultes ayant un } \\
\text { TSA. }\end{array}$ & $\begin{array}{l}\qquad \text { QT } \\
\text { Évaluation du statut } \\
\text { résidentiel actuel et } \\
\text { des facteurs de risque. }\end{array}$ & $\begin{array}{l}\text { Données issues } \\
\text { de la NLTS- } 2^{4} \\
\text { Personnes ayant } \\
\text { un TSA ( } N= \\
620 \text { ) âgées en } \\
\text { moyenne de } 23 \\
\text { ans. }\end{array}$ & $\begin{array}{l}\text { Les personnes ayant un TSA sont moins susceptibles } \\
\text { d'avoir déjà vécu de façon indépendante et plus enclines à } \\
\text { vivre sous surveillance parentale après l'école secondaire } \\
\text { comparativement à des personnes ayant des troubles } \\
\text { émotionnels ou d'apprentissage. Plusieurs d'entre elles } \\
\text { ignorent la possibilité d'explorer des options résidentielles } \\
\text { (FR). }\end{array}$ \\
\hline $\begin{array}{l}\text { Bossaert, } \\
\text { Colpin, Pijl et } \\
\text { Petry (2013) }\end{array}$ & $\begin{array}{l}\text { Étudier la PS } \\
\text { des élèves ayant } \\
\text { des besoins } \\
\text { particuliers. }\end{array}$ & $\begin{array}{l}\qquad \text { QT } \\
\text { Questionnaires } \\
\text { sociométriques pour } \\
\text { évaluer la PS. }\end{array}$ & $\begin{array}{l}\mathrm{N}=86 \text { élèves } \\
\text { ayant un TSA, } \\
61 \text { élèves ayant } \\
\text { une déficience } \\
\text { physique, } \\
1926 \text { élèves au } \\
\text { développement } \\
\text { typique }\end{array}$ & $\begin{array}{l}\text { La participation sociale est définie par les interactions } \\
\text { sociales, l'acceptation par les pairs, les amitiés et le concept } \\
\text { de soi social (l'identité) (définition). La PS des élèves ayant } \\
\text { un TSA est inférieure à celle des élèves au développement } \\
\text { typique et semblable à celle des élèves ayant un handicap } \\
\text { moteur et/ou sensoriel (FR). }\end{array}$ \\
\hline
\end{tabular}

4. La National Longitudinal Transition Study-2, 2001-2009 est une enquête nationale qui a été réalisée aux États-Unis et qui documente l'expérience vécue par des élèves lors de la transition de l'école à la vie active. 


\begin{tabular}{|c|c|c|c|c|}
\hline $\begin{array}{l}\text { Bottema- } \\
\text { Beutel et Li } \\
(2015)\end{array}$ & $\begin{array}{l}\text { Étudier les } \\
\text { justifications } \\
\text { d'adolescents au } \\
\text { développement } \\
\text { typique de ne } \\
\text { pas inclure } \\
\text { un pair avec } \\
\text { incapacités. }\end{array}$ & $\begin{array}{l}\quad \text { QL } \\
\text { Entrevues semi- } \\
\text { structurées pour } \\
\text { obtenir les jugements } \\
\text { et justifications. }\end{array}$ & $\begin{array}{l}\mathrm{N}=38 \\
\text { adolescents au } \\
\text { développement } \\
\text { typique âgés de } \\
13 \text { à } 18 \text { ans. }\end{array}$ & $\begin{array}{l}\text { Les adolescents évaluent l'exclusion des jeunes ayant } \\
\text { des incapacités de différentes façons selon le contexte : il } \\
\text { est inacceptable d'exclure un jeune de la classe ou d'une } \\
\text { activité de soccer spontanée (FP). Il est acceptable de ne } \\
\text { pas choisir un jeune ayant des incapacités pour un exercice } \\
\text { de laboratoire en classe ou de ne pas l'inviter à la maison; } \\
\text { expression de malaise et de sentiment d'incompétence des } \\
\text { pairs (FR). }\end{array}$ \\
\hline $\begin{array}{l}\text { Brock, Huber, } \\
\text { Carter, } \\
\text { Juarez et } \\
\text { Warren } \\
(2014)\end{array}$ & $\begin{array}{l}\text { Évaluer les } \\
\text { besoins de } \\
\text { perfectionne- } \\
\text { ment } \\
\text { professionnel } \\
\text { du personnel } \\
\text { travaillant avec } \\
\text { des élèves ayant } \\
\text { un TSA. }\end{array}$ & $\begin{array}{l}\qquad \text { QT } \\
\text { Évaluation du niveau } \\
\text { de confiance quant à } \\
\text { l'utilisation de pratiques } \\
\text { fondées sur des } \\
\text { données probantes }\end{array}$ & $\begin{array}{l}\mathrm{N}=456 \\
\text { intervenants } \\
\text { scolaires }\end{array}$ & $\begin{array}{l}\text { Dans l'ensemble, les enseignants ne sont pas très confiants } \\
\text { dans leur capacité à mettre en œuvre des pratiques fondées } \\
\text { sur des données probantes concernant les élèves ayant un } \\
\text { TSA (FR). } \\
\text { Le développement professionnel sur des pratiques fondées } \\
\text { sur des données probantes pour les élèves TSA est un besoin } \\
\text { critique (FR). } \\
\text { Les éducateurs sont plus à l'aise et expriment davantage } \\
\text { d'intérêt (FP). }\end{array}$ \\
\hline $\begin{array}{l}\text { Broderick, } \\
\text { Caswell, } \\
\text { Gregory, } \\
\text { Marzolini et } \\
\text { Wilson } \\
(2002)\end{array}$ & $\begin{array}{l}\text { Explorer les } \\
\text { possibilités } \\
\text { d'intégration } \\
\text { des adolescents } \\
\text { ayant un TSA } \\
\text { dans des } \\
\text { groupes et clubs. }\end{array}$ & $\begin{array}{l}\quad \text { QT } \\
\text { Les jeunes ayant un } \\
\text { TSA ont participé } \\
\text { à un groupe de } \\
\text { compétences sociales }\end{array}$ & $\begin{array}{l}\mathrm{N}=9 \\
\text { adolescents } \\
\text { âgés de } 12 \text { à } \\
15 \text { ans ayant } \\
\text { un TSA et } \\
\text { fréquentant } \\
\text { des écoles } \\
\text { régulières. }\end{array}$ & $\begin{array}{l}\text { Les adolescents ayant un TSA ont besoin de développer leurs } \\
\text { habiletés sociales (ex. : la communication, le contact visuel, la } \\
\text { posture du corps, l'expression et la compréhension de signes } \\
\text { non verbaux, la résolution de conflits et des techniques de } \\
\text { relaxation) (stratégies). } \\
\text { Le transfert des apprentissages (en groupe restreint) dans } \\
\text { des groupes réels permet d'augmenter la motivation en raison } \\
\text { de l'atteinte de l'objectif de façon concrète (FP/stratégies). }\end{array}$ \\
\hline
\end{tabular}

Brock, Huber, Évaluer les

Carter, besoins de perfectionneWarren ment travaillant avec des élèves ayant

Broderick, zolini ilso dans des groupes et clubs. obtenir les jugements et justifications.

$N=456$ intervenants de confiance quant à l'utilisation de pratiques ondées sur des données probantes

Les jeunes ayant un TSA ont participé à un groupe de compétences sociales des écoles
régulières. des groupes réels permet d'augmenter la motivation en raison de l'atteinte de l'objectif de façon concrète (FP/stratégies). 


$\begin{array}{lll}\text { Carter, } & \text { Étudier les } & \text { Recension des écrits } \\ \text { Harvey, } & \text { approches } & \text { scientifiques } \\ \text { Taylor et } & \text { efficaces pour } & \\ \text { Gotham } & \text { développer les } & \\ (2013) & \text { liens entre le } & \\ & \text { jeune ayant } & \\ & \text { un TSA et sa } & \\ & \text { communauté. }\end{array}$

Chen, Bundy, Étudier la

Cordier, motivation

Chien et à participer

Einfeld socialement

(2015) d'ados et jeunes adultes ayant un TSA.

\section{Chiang, Identifier les}

Cheung, Li et facteurs associés

Tsai

(2013)

\section{QT}

Les participants enregistraient leurs activités, avec qui, les difficultés perçues et la réciprocité sociale.

\section{QT}

Une analyse des données secondaires de la NLTS-2 a été réalisée. à la participation

à l'emploi pour

les finissants du

secondaire ayant

un TSA.

Adolescents et jeunes adultes ayant un TSA.

$\mathrm{N}=30$ personnes ayant proches ont été associées positivement à une plus grande un TSA âgées de autodétermination (FP).

16 à 45 ans. Les activités sociales et les interactions avec les amis facilitent la motivation à s'engager socialement (FP). Les difficultés perçues peuvent être dues à des expériences personnelles antérieures négatives (être exclu) (FR).

Données issues de la NLTS-2.

$\mathrm{N}=830$ élèves

âgés de 13 à 16 ans et ayant un TSA

Les compétences sociales, la diplomation au secondaire quant au développement vocationnel du jeune sont des Les difficultés de communication sociale et les modèles
Vivre des expériences significatives à l'école secondaire pour qualifications pour faire un choix éclairé pour l'avenir (FP). II est important de planifier la transition selon les intérêts, les compétences et les besoins du jeune avant la dernière année de scolarisation au secondaire (FP/stratégies).

Les familles des jeunes ayant un TSA expriment des difficultés à trouver l'information nécessaire à l'intégration communautaire (FR). l'opportunité d'avoir des conseils et la mobilisation de l'école facteurs importants associés à la participation à l'emploi (FP). restreints et répétitifs de comportement peuvent affecter leurs chances d'obtenir des emplois rémunérés (FR). 


\begin{tabular}{|c|c|c|c|c|}
\hline $\begin{array}{l}\text { Crabtree } \\
(2007)\end{array}$ & $\begin{array}{l}\text { Étudier les } \\
\text { perceptions des } \\
\text { mères quant à } \\
\text { l'acceptation } \\
\text { de la famille } \\
\text { et l'inclusion } \\
\text { sociale. }\end{array}$ & $\begin{array}{l}\quad \text { QL } \\
\text { Entrevues semi- } \\
\text { structurées }\end{array}$ & $\begin{array}{l}\mathrm{N}=15 \text { mères } \\
\text { d'enfants (4 à } \\
16 \text { ans) ayant } \\
\text { une déficience } \\
\text { intellectuelle ou } \\
\text { développemen- } \\
\text { tale (autisme). }\end{array}$ & $\begin{array}{l}\text { La sensibilisation de la population aux différences et } \\
\text { handicaps réduit la ségrégation et augmente l'acceptation } \\
\text { (stratégies/FP). } \\
\text { Les parents doivent avoir un bon lien avec les intervenants } \\
\text { (FP). } \\
\text { L'acquisition de la parole et l'apprentissage de comportements } \\
\text { sociaux appropriés favorisent l'acceptation de l'enfant au sein } \\
\text { de la communauté (FP/stratégies). }\end{array}$ \\
\hline $\begin{array}{l}\text { Eaves et Ho } \\
(2008)\end{array}$ & $\begin{array}{l}\text { Étude } \\
\text { longitudinale } \\
\text { des réalisations } \\
\text { des personnes } \\
\text { ayant un TSA en } \\
\text { termes de travail, } \\
\text { d'amitiés et } \\
\text { d'autonomie. }\end{array}$ & $\begin{array}{l}\qquad \text { QT } \\
\text { Étude longitudinale en } \\
\text { trois temps. }\end{array}$ & $\begin{array}{l}\mathrm{N}=48 \\
\text { participants } \\
\text { ayant un TSA. } \\
\text { Âge moyen au } \\
\text { temps } 1: 6,8 \\
\text { ans; temps } 2: \\
11,4 \text { ans; temps } \\
3: 24 \text { ans. }\end{array}$ & $\begin{array}{l}\text { Une conséquence de la connaissance de ses propres } \\
\text { limitations sociales peut être que les adolescents ayant un } \\
\text { TSA deviennent frustrés par leurs déficits sociaux, ce qui peut } \\
\text { les conduire à moins de participation et d'engagement dans } \\
\text { des activités sociales (FR). } \\
\text { Les adolescents et jeunes adultes identifient des besoins } \\
\text { sur le plan du travail, des activités sociales et des relations } \\
\text { amicales (FR). }\end{array}$ \\
\hline $\begin{array}{l}\text { Farley, } \\
\text { McMahon, } \\
\text { Fombonne, } \\
\text { Jenson et al. } \\
\text { (2009) }\end{array}$ & $\begin{array}{l}\text { Définir les } \\
\text { besoins et } \\
\text { la fonction } \\
\text { des services } \\
\text { actuellement } \\
\text { offerts. }\end{array}$ & $\begin{array}{l}\qquad \text { QT } \\
\text { Évaluation } \\
\text { diagnostique et globale } \\
\text { à deux moments. }\end{array}$ & $\begin{array}{l}\mathrm{N}=41 \\
\text { personnes ayant } \\
\text { un TSA. } \\
\text { Temps } 1: 7,2 \\
\text { ans et temps } 2: \\
32,5 \text { ans. }\end{array}$ & $\begin{array}{l}\text { Malgré des taux d'intégration en emploi rémunéré de } 20 \text { à } \\
55 \% \text {, la majorité des personnes ayant un TSA travaillent dans } \\
\text { des ateliers protégés ou des centres de jour (FR). } \\
\text { Plusieurs jeunes développent des états dépressifs et anxieux } \\
\text { (FR). } \\
\text { Des compétences dans les AVQ prédiraient le succès de la } \\
\text { transition et l'autonomie chez les adolescents ayant un TSA } \\
\text { (FP). }\end{array}$ \\
\hline
\end{tabular}




\begin{tabular}{|c|c|c|c|c|}
\hline $\begin{array}{l}\text { Freeth, } \\
\text { Bullock et } \\
\text { Milne } \\
(2013)\end{array}$ & $\begin{array}{l}\text { Évaluer la } \\
\text { prévalence et } \\
\text { la corrélation } \\
\text { entre les traits } \\
\text { associés à } \\
\text { l'anxiété sociale } \\
\text { et l'autisme. }\end{array}$ & $\begin{array}{l}\text { QT } \\
\text { Les traits associés à } \\
\text { l'autisme et l'anxiété } \\
\text { sociale ont été évalués. }\end{array}$ & $\begin{array}{l}\mathrm{N}=1325 \\
\text { étudiants âgés } \\
\text { de } 20 \text { ans en } \\
\text { moyenne. }\end{array}$ & $\begin{array}{l}\text { Les étudiants ayant des niveaux élevés de traits autistiques } \\
\text { sont plus susceptibles d'éprouver une grande anxiété sociale } \\
\text { que ceux ayant des niveaux moyens ou faibles de traits } \\
\text { autistiques (FR). } \\
\text { Les étudiants ayant un TSA ont besoin de développer } \\
\text { leurs habiletés sociales afin de poursuivre des études } \\
\text { postsecondaires puisque ces habiletés sont requises dans } \\
\text { ces environnements (stratégies). }\end{array}$ \\
\hline $\begin{array}{l}\text { Goldingay, } \\
\text { Stagnitti, } \\
\text { Sheppard, } \\
\text { McGillivray, } \\
\text { McLean et } \\
\text { Pepin (2015) }\end{array}$ & $\begin{array}{l}\text { Expérimenter } \\
\text { une intervention } \\
\text { de jeux } \\
\text { d'imagination } \\
\text { pour la } \\
\text { compréhension } \\
\text { sociale d'ados } \\
\text { ayant un TSA }\end{array}$ & $\begin{array}{l}\qquad \text { QT } \\
\text { Évaluation pré et } \\
\text { post-intervention. } \\
\text { Évaluations aussi par } \\
\text { les parents }\end{array}$ & $\begin{array}{l}\mathrm{N}=5 \\
\text { adolescents } \\
\text { ayant un TSA } \\
\text { âgé de } 13,5 \text { ans } \\
\text { en moyenne. }\end{array}$ & $\begin{array}{l}\text { Le jeu imaginaire soutient le développement de compétences } \\
\text { de base pour le développement d'interactions sociales } \\
\text { complexes (FP/stratégies). } \\
\text { Suite à l'intervention, des améliorations ont été observées } \\
\text { dans la flexibilité de la pensée et le comportement social. } \\
\text { L'autorégulation a également augmenté, selon les parents } \\
\text { (FP). }\end{array}$ \\
\hline $\begin{array}{l}\text { Gray, } \\
\text { Keating, } \\
\text { Taffe, } \\
\text { Brereton, } \\
\text { Einfeld, } \\
\text { Reardon et } \\
\text { Tonge } \\
(2014)\end{array}$ & $\begin{array}{l}\text { Analyser } \\
\text { l'inclusion } \\
\text { communautaire } \\
\text { et les } \\
\text { compétences } \\
\text { dans les } \\
\text { activités de vie } \\
\text { quotidienne }\end{array}$ & $\begin{array}{l}\quad \text { QT } \\
\text { Entrevues et passation } \\
\text { d'instruments de } \\
\text { mesure. }\end{array}$ & $\begin{array}{l}119 \text { participants } \\
\text { au temps } 1,89 \\
\text { au temps } 5 . \\
\text { Âge moyen : } \\
\text { temps } 1: 8,7 \\
\text { ans et temps } 2: \\
24,8 \text { ans. }\end{array}$ & $\begin{array}{l}\text { Globalement, le premier constat est un degré élevé de } \\
\text { dépendance, soit à des familles ou à des services de soutien } \\
\text { (FR). } \\
\text { Les compétences dans les activités de vie quotidienne (AVQ) } \\
\text { sont un aspect important de l'intégration communautaire, en } \\
\text { particulier en ce qui concerne la vie autonome, le travail et } \\
\text { l'engagement social dans la communauté (FP). }\end{array}$ \\
\hline
\end{tabular}




\section{Hagner,}

Kurtz,

Cloutier,

Arakelian,

Brucker et

May

(2012)

Hart, Grigal

et Weir

(2010)

\section{Aperçu des \\ options \\ d'éducation \\ postsecondaire \\ pour les \\ étudiants ayant \\ un TSA ou une \\ DI.}

Hendricks et La transition de

Wehman

(2009)

l'école à la vie

active pour les

jeunes ayant un

TSA.

Huws et

Étudier les

Jones (2015) perceptions de

l'autisme de

jeunes ayant un

TSA.
$N=47$ jeunes

ayant un TSA

âgés de 16 à 19

ans.

transition pour un

groupe et un deuxième

groupe à l'année 2

Recension des écrits scientifiques

Recension des écrits scientifiques

\section{QL}

Entrevues semistructurées un TSA.

$\mathrm{N}=9$ jeunes ayant un diagnostic de
La capacité d'interagir avec les autres et communiquer ses préférences est nécessaire pour participer à la planification de la transition (FR).

Plusieurs facteurs externes peuvent atténuer l'effet des symptômes liés au TSA : attentes des éducateurs et des parents (ne pas les réduire); qualité de l'éducation et de la PS dans la communauté, expériences de travail; qualité du plan de transition; taille du réseau social et capacité d'accéder au soutien requis pour la PS (FP).

Les personnes Les personnes doivent connaitre les différents services offerts ayant un TSA et/ dans leur communauté afin de pouvoir les exploiter (FPI ou une DI. stratégies).

Les intervenants scolaires doivent connaitre et discuter des possibilités de faire des études postsecondaires avec les personnes ayant un TSA ou une DI et leur famille (FR).

Les jeunes ayant Les élèves ayant un TSA ont besoin que l'équipe de professionnels intervienne dans le même sens pour les aider à réaliser leur projet de vie (FP/stratégies).

Pour les adolescents ayant un TSA, l'éducation et la formation doivent être faites dans la communauté pendant l'école secondaire (FP). Les adolescents ayant un syndrome d'Asperger sont conscients de leurs différences (FP). En interagissant avec les membres de leur entourage et leurs pairs, ils ont appris à être TSA, âgés de 16 plus habiles socialement et cela leur a permis d'améliorer leur à 21 ans. autonomie et leur indépendance (FP). 


\begin{tabular}{|c|c|c|c|c|}
\hline $\begin{array}{l}\text { Jones et } \\
\text { Frederickson } \\
(2010)\end{array}$ & $\begin{array}{l}\text { Étudier les } \\
\text { caractéristiques } \\
\text { comportemen- } \\
\text { tales prédictives } \\
\text { d'une intégration } \\
\text { réussie dans } \\
\text { l'enseignement } \\
\text { régulier. }\end{array}$ & $\begin{array}{l}\quad \text { QT } \\
\text { Évaluation du } \\
\text { fonctionnement } \\
\text { intellectuel, de } \\
\text { l'inclusion sociale, des } \\
\text { forces et des difficultés, } \\
\text { des habiletés sociales } \\
\text { et de l'intimidation. }\end{array}$ & $\begin{array}{l}\mathrm{N}=86 \text { élèves } \\
\text { âgés en } \\
\text { moyenne de } \\
11 \text { ans dont } \\
43 \text { avaient un } \\
\text { diagnostic de } \\
\text { TSA et } 43 \text { étaient } \\
\text { dans le groupe } \\
\text { témoin. }\end{array}$ & $\begin{array}{l}\text { Les résultats suggèrent aux écoles d'améliorer leurs } \\
\text { programmes de développement des habiletés sociales } \\
\text { pour les jeunes ayant un TSA et de tenir des activités de } \\
\text { sensibilisation auprès des élèves réguliers, au sujet des } \\
\text { élèves ayant un TSA dans les classes ordinaires (stratégies/ } \\
\text { FP). }\end{array}$ \\
\hline $\begin{array}{l}\text { Kreider, } \\
\text { Bendixen, } \\
\text { Mann, Young } \\
\text { et McCarty } \\
(2015)\end{array}$ & $\begin{array}{l}\text { Identifier } \\
\text { et décrire } \\
\text { les facteurs } \\
\text { contributifs à } \\
\text { la participation } \\
\text { dans les réseaux } \\
\text { sociaux. }\end{array}$ & $\begin{array}{l}\text { Mixte } \\
\text { Élaboration d'une carte } \\
\text { du réseau social de } \\
\text { l'adolescent } \\
\text { et entrevue avec lui }\end{array}$ & $\begin{array}{l}\mathrm{N}=36 \text { jeunes } \\
\text { de } 11 \text { à } 16 \text { ans } \\
\text { ayant un TSA, } \\
\text { TDAH ou DA. } \\
\mathrm{N}=17 \text { jeunes, } \\
\text { même âge au } \\
\text { développement } \\
\text { typique }\end{array}$ & $\begin{array}{l}\text { Plus le jeune a des amis, plus il réalisera des activités avec } \\
\text { d'autres personnes que les membres de sa famille (FP). } \\
\text { Il y aurait une corrélation forte et positive entre la } \\
\text { diversification des activités réalisées et le niveau de soutien } \\
\text { de la part de ses amis (FP). }\end{array}$ \\
\hline $\begin{array}{l}\text { Kuo, } \\
\text { Orsmond, } \\
\text { Cohn et } \\
\text { Coster } \\
(2013)\end{array}$ & $\begin{array}{l}\text { Recueillir des } \\
\text { données auprès } \\
\text { des adolescents } \\
\text { ayant un TSA } \\
\text { afin d'explorer } \\
\text { les activités } \\
\text { qu'ils réalisent } \\
\text { avec leurs amis. }\end{array}$ & $\begin{array}{l}\quad \text { QT } \\
\text { Les participants et } \\
\text { leurs parents ont } \\
\text { rempli un questionnaire } \\
\text { identifiant les activités } \\
\text { réalisées avec des } \\
\text { amis. }\end{array}$ & $\begin{array}{l}\mathrm{N}=91 \text { jeunes } \\
\text { ayant un TSA } \\
\text { âgés de } 12 \text { à } 18 \\
\text { ans. }\end{array}$ & $\begin{array}{l}\text { L'adolescence peut être une période particulièrement difficile } \\
\text { pour les adolescents ayant un TSA puisque l'environnement } \\
\text { social devient plus complexe et ceux-ci deviennent plus } \\
\text { conscients de leurs difficultés sociales (FR). } \\
\text { Presque tous les adolescents ayant un TSA (96\%) ont dit } \\
\text { avoir au moins un ami (FP). }\end{array}$ \\
\hline
\end{tabular}




$\begin{array}{ll}\text { Liptak, } & \text { Décrire la PS } \\ \text { Kennedy et } & \text { et identifier les } \\ \text { Dosa } & \text { facteurs qui } \\ \text { (2011) } & \text { l'influencent. }\end{array}$

Myers, Davis, Étudier la PS et Stobbe et Bjornson (2015)

Attridge,

(2015)

ansition de l'âge adulte.
Une analyse des données secondaires de la NLTS-2 a été réalisée.
$\mathrm{N}=725$ jeunes ayant un TSA.

Âge moyen : temps $1: 15,4$ ans et temps 2 : 19,2 ans
$55,4 \%$ des adolescents ayant un TSA n'ont jamais réalisé d'activités sociales avec un ami et $63,9 \%$ n'ont jamais reçu d'appel téléphonique de la part d'un ami dans les 12 mois précédant l'enquête (FR).

La capacité de communiquer efficacement, les symptômes autistiques moins sévères et avoir des parents présents ont été associés à des résultats plus positifs de PS (FP).

Les services offerts aux personnes doivent être individualisés et contextualisés pour répondre à leurs besoins (stratégies).

Données issues L'adolescence peut être une période privilégiée pour établir de la NLTS-2. $\mathrm{N}=17818$ élèves âgés de 13 à 16 ans et

ayant un TSA.

l'adolescence à données secondaire de la NLTS-2 a été réalisée.

Recension des écrits $\quad N=10$ études scientifiques des liens avec les personnes de la communauté (FP).

La PS est tributaire de la capacité inhérente de la personne à communiquer et à interagir avec les autres (FP).

La recherche démontre que de l'adolescence à l'âge adulte, les personnes ayant un TSA comptent sur les autres pour les aider à planifier des opportunités communautaires et sociales (FR).

Plus l'emploi est compétitif, plus les compétences adaptatives des individus sont renforcées, ce qui suggère que l'emploi concurrentiel peut améliorer la capacité d'un individu à s'intégrer dans la communauté (FP).

Les adolescents et les jeunes adultes ayant un TSA doivent développer leurs compétences de communication interpersonnelle et d'habiletés de travail, avec du soutien de la part d'un « coach », afin d'acquérir une stabilité d'emploi (FP). 


\section{Orsmond,}

Shattuck,

Cooper,

Sterzing et

Anderson

(2013)

\section{Penney}

(2013)

\section{Étudier la PS de jeunes adultes ayant un TSA.}

\section{QT}

Une analyse des données secondaire de la NLTS-2 a été réalisée.

\section{Explorer}

l'expérience des personnes ayant un TSA qui ont aussi développé une dépression et/ou un trouble d'anxiété associé.

Données issues Les jeunes adultes ayant un TSA étaient significativement de la NLTS-2.

$\mathrm{N}=400$

participants

âgés de 21 à 25

ans qui avaient

un diagnostic

de TSA, de DI,

de difficultés

émotionnelles

ou de difficultés

d'apprentissage.

plus susceptibles de ne jamais voir des amis, ne jamais être appelés par des amis, ne jamais être invités à des activités et d'être socialement isolés comparativement aux autres groupes de comparaison (FR).

Pour les personnes ayant un TSA, la difficulté à converser et de faibles compétences fonctionnelles étaient prédicteurs d'une moindre PS (FR).

Des interventions doivent être mises en place afin de soutenir les habiletés sociales et la communication de ces jeunes en plus de soutenir la famille et les milieux afin d'offrir des opportunités sociales au jeune adulte (stratégies).

$\mathrm{N}=5$

adolescents ou jeunes adultes (âgés de 16 à 21 ans) ayant un TSA.

$\mathrm{N}=10$ mères

d'adolescent ou jeune adulte (âgés de 15 à 21 ans) ayant un TSA.
Cette étude met en lumière le fait que les adolescents et jeunes adultes croient que les enseignants ne sont pas suffisamment formés pour faire face à l'apprentissage, aux particularités sensorielles, et aux problèmes de comportement associés à l'autisme (FR).

Les connaissances des enseignants sur le TSA influencent le succès de ces élèves dans la classe (FP).

Les parents ont mentionné la nécessité de travailler en collaboration, d'avoir une relation ouverte et digne de confiance et d'être capables de communiquer régulièrement avec le milieu scolaire (FP). 


\begin{tabular}{|c|c|c|c|c|}
\hline $\begin{array}{l}\text { Schall, } \\
\text { Cortijo-Doval, } \\
\text { Sherron } \\
\text { Targett et } \\
\text { Wehman } \\
(2006)\end{array}$ & $\begin{array}{l}\text { Identifier les } \\
\text { stratégies } \\
\text { permettant } \\
\text { de soutenir la } \\
\text { transition vers } \\
\text { la vie active des } \\
\text { personnes ayant } \\
\text { un TSA. }\end{array}$ & Chapitre de livre & $\begin{array}{l}\text { Les personnes } \\
\text { ayant un TSA } \\
\text { en processus de } \\
\text { transition vers la } \\
\text { vie active. }\end{array}$ & $\begin{array}{l}\text { Les différents intervenants gravitant autour de la personne } \\
\text { doivent se concerter quant aux objectifs à atteindre (FP). } \\
\text { Les personnes ayant un TSA doivent développer leurs } \\
\text { habiletés sociales (FP/stratégies). } \\
\text { La présence et l'implication de la famille lors de la transition } \\
\text { sont primordiales (FP). } \\
\text { Pour faciliter le transfert des compétences acquises dans } \\
\text { des environnements différents, les enseignants devraient } \\
\text { permettre à l'élève d'avoir de multiples occasions d'apprendre } \\
\text { et de pratiquer les compétences dans différents contextes et } \\
\text { avec des gens différents (stratégies). }\end{array}$ \\
\hline $\begin{array}{l}\text { Shattuck, } \\
\text { Orsmond, } \\
\text { Wagner et } \\
\text { Cooper } \\
(2011)\end{array}$ & $\begin{array}{l}\text { Estimer les taux } \\
\text { de PS chez les } \\
\text { adolescents } \\
\text { ayant un TSA. }\end{array}$ & $\begin{array}{l}\quad \text { QT } \\
\text { Une analyse des } \\
\text { données secondaire } \\
\text { de la NLTS-2 a été } \\
\text { réalisée. }\end{array}$ & $\begin{array}{l}\text { Données issues } \\
\text { de la NLTS- } 2 \text {. } \\
\mathrm{N}=900 \text { élèves } \\
\text { âgés de } 13 \text { à } 16 \\
\text { ans et ayant un } \\
\text { TSA. }\end{array}$ & $\begin{array}{l}\text { Les résultats indiquent que la moitié des jeunes ayant } \\
\text { un TSA ont une expérience nulle ou très limitée dans la } \\
\text { réalisation d'activités sociales avec les amis et seulement un } \\
\text { tiers des participants réalisent des activités sociales dans la } \\
\text { communauté avec des pairs (FR). } \\
\text { Les jeunes ayant un TSA sont plus enclins à être bénévoles } \\
(35,1 \% \text { ) ou à suivre des cours à l'extérieur de l'école (30,6 \%) } \\
\text { que de réaliser des activités sociales (FP). } \\
\text { Pour améliorer les relations sociales, il s'avère important de } \\
\text { favoriser la participation des jeunes ayant un TSA avec leurs } \\
\text { pairs dans des activités de groupes (stratégies). } \\
\text { Les difficultés langagières et la faible compétence dans la } \\
\text { communication sociale sont associées à une moindre PS } \\
\text { (FR). } \\
\text { Favoriser les interventions réalisées en contexte naturel } \\
\text { (stratégies). }\end{array}$ \\
\hline
\end{tabular}

Shattuck Orsmond, Wagner et Coope (2011)

Estimer les taux adolescents ayant un TSA de la NLTS-2 a été réalisée.
Données issues âgés de 13 à 16 ans et ayant un TSA 


\begin{tabular}{|c|c|c|c|c|}
\hline $\begin{array}{l}\text { Taylor et } \\
\text { Seltzer (2011) }\end{array}$ & $\begin{array}{l}\text { Décrire les } \\
\text { activités } \\
\text { professionnelles } \\
\text { et de jour pour } \\
\text { un groupe de } \\
\text { jeunes adultes } \\
\text { ayant un TSA } \\
\text { qui avaient } \\
\text { quitté l'école } \\
\text { secondaire. }\end{array}$ & $\begin{array}{l}\qquad \text { QT } \\
\text { Évaluation des } \\
\text { activités de jour, de } \\
\text { troubles associés, } \\
\text { du revenu familial, } \\
\text { de la sévérité des } \\
\text { symptômes autistiques, } \\
\text { des comportements } \\
\text { mésadaptés, de } \\
\text { l'indépendance } \\
\text { fonctionnelle et de } \\
\text { troubles mentaux en } \\
\text { comorbidité. }\end{array}$ & $\begin{array}{l}\mathrm{N}=66 \\
\text { personnes ayant } \\
\text { un diagnostic de } \\
\text { TSA âgées en } \\
\text { moyenne de } 23 \\
\text { ans. }\end{array}$ & $\begin{array}{l}\text { Des indices du fonctionnement comportemental tels que de } \\
\text { faibles symptômes autistiques, l'absence de comportements } \\
\text { inadaptés, des compétences fonctionnelles et l'absence de } \\
\text { troubles psychiatriques concomitants sont liés aux activités } \\
\text { d'emploi des jeunes adultes ayant un TSA dans les années } \\
\text { suivant la sortie de l'école secondaire (FP). } \\
\text { Les jeunes adultes qui fréquentent un programme } \\
\text { d'enseignement postsecondaire ou étaient dans des emplois } \\
\text { concurrentiels ont moins de symptômes/comportements } \\
\text { autistiques et une plus grande indépendance fonctionnelle } \\
\text { (FP). }\end{array}$ \\
\hline $\begin{array}{l}\text { Test, Smith et } \\
\text { Carter } \\
(2014)\end{array}$ & $\begin{array}{l}\text { Étudier les } \\
\text { aspects } \\
\text { importants de } \\
\text { l'éducation pour } \\
\text { une transition } \\
\text { réussie à la vie } \\
\text { active chez les } \\
\text { adolescents } \\
\text { ayant un TSA. }\end{array}$ & $\begin{array}{l}\text { Recension des écrits } \\
\text { scientifiques }\end{array}$ & $\begin{array}{l}\text { Les adolescents } \\
\text { et jeunes adultes } \\
\text { ayant un TSA. }\end{array}$ & $\begin{array}{l}\text { Les élèves ayant un TSA doivent avoir accès à des } \\
\text { possibilités d'apprentissage qui ont une pertinence immédiate } \\
\text { et à long terme pour leur vie et qui favorisent des relations } \\
\text { valorisantes avec des membres clés de leur école et de leur } \\
\text { communauté (FP). } \\
\text { Il est essentiel que les étudiants aient des occasions } \\
\text { d'acquérir des compétences de carrière et des connaissances } \\
\text { à l'école (FP). } \\
\text { Ils doivent développer leur autodétermination et leurs } \\
\text { compétences sociales afin de pouvoir développer des } \\
\text { relations significatives avec leurs pairs (FP/stratégies). } \\
\text { Les milieux scolaires qui travaillent en partenariat avec les } \\
\text { organismes gouvernementaux et communautaires s'assurent } \\
\text { du suivi des interventions auprès du jeune et de sa famille } \\
\text { (FP/stratégies). }\end{array}$ \\
\hline
\end{tabular}


Tobin, Drager Étudier la PS et Richardson et le soutien

(2014) permettant de faciliter la PS des adultes ayant un TSA.

Wang et Berg Évaluer la (2014) participation des jeunes adultes ayant un TSA et explorer les facteurs qui entravent leur participation.
Recension des écrits scientifiques

$N=13$ articles

Travailler à améliorer les compétences sociales est d'une importance capitale puisque le fonctionnement social a un impact sur la PS dans une variété de domaines de la vie courante (FP).

La PS des personnes ayant un TSA semble être facilitée par le soutien informel, la participation à des groupes d'apprentissage de compétences sociales et l'appartenance à des groupes de soutien (FP).

Le soutien familial, des facteurs individuels (bonnes habiletés sociales) et des niveaux de sévérité plus faibles des symptômes de TSA ont été liés à des niveaux plus élevés de PS (FP).

Les résultats de l'étude mettent en lumière le fait que les adolescents et les jeunes adultes ayant un TSA préfèrent les activités sans interactions sociales (FR).

Évaluation de la participation des jeunes ayant un TSA lors de la transition vers la vie active.

$\mathrm{N}=11$ jeunes adultes ayant un TSA et leurs parents.

$\mathrm{N}=11$ jeunes adultes au développement typique

Les participants sont âgés de 18 à 25 ans.
Les obstacles nommés par les jeunes ayant un TSA quant à la participation à des activités sociales sont le manque de ressources personnelles et environnementales et l'absence de pair avec qui réaliser ces activités (FR).

Légende : QT : méthodologie quantitative; QL : méthodologie qualitative; FR : facteurs de risque; FP : facteurs de protection; PS : participation sociale. 
à l'adversité, alors que la personne reçoit de l'aide de l'entourage, de tuteurs de résilience ou de réseaux sociaux (Ionescu, 2016). Cette capacité psychologique se développe dès la petite enfance et pendant toute la vie. En raison de leur condition, les adolescents ou jeunes adultes ayant un TSA ont été confrontés au risque, à l'adversité et ont eu l'occasion de développer leur résilience naturelle. Ces pratiques favorisent leur participation sociale dans les différents contextes de vie : familial, scolaire, social, professionnel ou communautaire.

Favoriser le développement de compétences sociales ${ }^{5}$ et communicationnelles. La capacité à communiquer efficacement (Andersen, Shattuck, Cooper, Roux et Wagner, 2014; Crabtree, 2007; Eaves et Ho, 2008; Liptak et al., 2011; Tobin et al., 2014), les interactions positives du jeune ayant un TSA avec des amis proches (Chen, Bundy, Cordier, Chien et Einfeld, 2015; Kreider, Bendixen, Mann, Young et McCarty, 2015; Kuo et al., 2013) ainsi que le développement des compétences sociales (Chiang et al., 2013; Crabtree, 2007; Hendricks et Wehman, 2009; Myers et al., 2015; Penney, 2013; Tobin et al., 2014) sont considérés par plusieurs auteurs comme essentielles à la participation sociale. Ainsi dans leur recension des écrits, Carter et al. (2013) affirment que le développement de relations amicales et interpersonnelles est d'une importance capitale pour ces jeunes. Dans le contexte scolaire québécois, "socialiser " fait d'ailleurs partie intégrante du mandat des milieux scolaires (Gouvernement du Québec, 2006). Dans l'étude de Penney (2013), les participants mentionnent le besoin de développer et de mettre en pratique les compétences sociales. Ces dernières leur permettant de vivre des expériences positives lors de la scolarisation et d'avoir une meilleure estime de soi. II s'avère donc prioritaire de permettre à l'élève ayant un TSA de développer ses compétences sociales afin de mieux interagir à l'école et dans les milieux communautaires qu'il fréquente pendant et après sa scolarisation. Les résultats de l'étude de Huws et Jones (2015) appuient ceux de la recension des écrits de Carter et al. (2013). En effet, dans l'étude de Huws et Jones (2015), les participants se sont exprimés sur le bien-fondé du développement de leurs habiletés sociales en mentionnant qu'au fil des ans, l'inclusion dans les milieux réguliers leur a permis de s'adapter et d'être plus habiles socialement. Ainsi, les jeunes ayant un TSA reconnaissent l'importance de développer des relations sociales et interpersonnelles (Huws et Jones, 2015). Qui plus est, Schall et al. (2006) évoquent l'importance pour les adolescents de développer leurs habiletés sociales (communication, contact visuel, posture du corps, expressions figurées, compréhension des signes non verbaux, résolution de conflits, régulation). Pour ce faire, l'apprentissage des habiletés de communication devrait être amorcé dès l'enfance (Liptak et al., 2011). En effet, le jeune qui a développé ses habiletés de communication pourrait interagir plus facilement et socialiser avec son entourage et sa collectivité, réduisant les barrières à sa participation sociale actuelle et future.

5. Les compétences sociales incluent, dans une situation sociale donnée, la perception correcte des éléments inhérents à la situation (compétences "réceptives »), la recherche optimale des alternatives possibles (compétences de " traitement de l'information ») et l'adoption des comportements d'affirmation de soi (compétences « motrices ») (PsychiatrieConseil international de la langue française, 2017, cité dans http://www.psychologies.com/ Dico-Psycho/Competences-sociales). 
Favoriser des activités qui permettent le développement de la compréhension sociale. Plusieurs auteurs abordent les caractéristiques inhérentes au fonctionnement de la personne ayant un TSA (dont un déficit cliniquement significatif et persistant de la communication sociale) comme des entraves à sa participation sociale. Des difficultés reliées à l'empathie, l'autorégulation et la flexibilité de la pensée sont des caractéristiques des adolescents ayant un TSA et ces déficits représentent des obstacles importants au développement et au maintien de relations sociales (Goldingay et al., 2015). La mise en place d'activités qui permettent d'améliorer la compréhension sociale est donc pertinente afin de contrer ces obstacles. Sur ce plan, l'étude de Goldingay et al. (2015), réalisée auprès de cinq adolescents ayant un diagnostic de TSA (âge moyen : 13,5 ans), a permis de mettre en évidence des stratégies pour faciliter leur compréhension sociale. Ainsi, les jeux de rôle à partir de mises en situation fictives ont permis d'améliorer la régulation émotionnelle des participants et de développer leurs capacités de résolution de problèmes. En effet, les jeux de rôles soutiennent le développement de l'interaction réciproque soutenue, la compréhension narrative, la compréhension des rôles, la flexibilité de la pensée, la métacognition et la résolution de problèmes, des compétences de base pour la poursuite du développement dans les interactions sociales complexes (Goldingay et al., 2015). De plus, l'utilisation de jeux de rôles pour favoriser le développement de la compréhension sociale peut être mis en place dans le milieu scolaire, pour enrichir et diversifier les activités proposées. De la même façon, les activités sociales ou de loisirs dans la collectivité peuvent devenir des occasions en contexte naturel d'expérimenter des jeux de rôles et autres stratégies favorisant la compréhension sociale.

Favoriser les apprentissages en contexte naturel. Un défi pour les personnes ayant un TSA est de transférer et de généraliser des apprentissages dans divers contextes (Brown et Bebko, 2012). Ainsi, un facteur de protection d'importance est la participation à des activités dans la communauté, telles que le bénévolat (Orsmond, Krauss et Seltzer, 2004; Shattuck et al., 2011; Taylor et Seltzer, 2011), les activités sociales (Chen et al., 2015; Kreider et al., 2015; Wang et Berg, 2014), les activités parascolaires (Carter et al., 2013) et l'intégration d'un milieu de travail (Gray et al., 2014; Nicholas, Attridge, Zwaigenbaum et Clarke, 2015; Taylor et Seltzer, 2011; Wang et Berg, 2014). En raison des difficultés liées à leur condition, notamment la faible compréhension globale et les problèmes de généralisation des apprentissages (Brown et Bebko, 2012), plusieurs auteurs soulignent l'importance des apprentissages contextualisés (Hart et al., 2010; Liptak et al., 2011; Schall et al., 2006; Shattuck et al., 2011) tout particulièrement pour développer des habiletés sociales (Carter et al., 2013; Chen et al., 2015; Freeth, Bullock et Milne, 2013; Jones et Frederickson, 2010; Schall et al., 2006). Dans leurs travaux, Schall et al. (2006) mentionnent que l'apprentissage et la mise en pratique de leurs compétences dans différents contextes et avec des gens différents permettent de faciliter le transfert de ces compétences dans les milieux fréquentés par les jeunes ayant un TSA. De plus, les apprentissages en contexte naturel favorisent une meilleure participation sociale en leur permettant d'apprivoiser et de développer leurs connaissances des environnements dans lesquels ils évolueront à la fin de la scolarisation. 
Favoriser la participation à des groupes de développement des compétences sociales. L'étude de Broderick et al. (2002) vise à explorer les possibilités permettant de soutenir les adolescents ayant un TSA dans leur intégration au sein d'un groupe social. Les participants $(N=9)$ sont âgés de 12 à 15 ans et fréquentent des écoles ordinaires. Ils ont participé à un programme de développement des compétences sociales avec des mentors et les compétences apprises ont ensuite été transférées dans des groupes réels d'activités où les mentors leur ont donné de la rétroaction sur leur comportement. Les jeunes ont aussi participé à des séances supplémentaires de développement des compétences sociales durant la semaine précédant le début de leur club (groupe réel) et dans la semaine suivante. Des questionnaires ont été remplis par les jeunes, ainsi que par leurs familles, leurs professeurs et leurs mentors pour mesurer les compétences sociales. Il a été observé que l'estime de soi (mesurée à l'aide de l'échelle PierreHarris Children's Self Concept Scale) des adolescents avait augmenté entre le début et la fin du projet en raison de l'application concrète des apprentissages dans des milieux réels. Ces résultats soulignent la pertinence d'enrichir la formation qui vise le développement des habiletés sociales de ces adolescents, en planifiant le transfert des habiletés acquises dans des groupes sociaux réels. Les adolescents peuvent ainsi consolider leurs compétences sociales avec leurs pairs et des mentors, partager une expérience de loisirs en groupe et développer un sentiment d'appartenance à leur collectivité.

Favoriser le développement de l'autodétermination. Test et al. (2014) mettent en évidence l'importance de l'autodétermination pour la participation sociale. Leur recension des écrits permet de préciser que le développement de l'autodétermination inclut plusieurs compétences liées à la défense des droits, à la prise de décision, à l'établissement d'objectifs et à la résolution de problèmes. Dans l'étude de Chen et al. (2015), les interactions avec les membres de la famille et les amis proches ont été associées positivement à une plus grande autodétermination. Les participants de cette étude étaient plus susceptibles de développer leur autodétermination lorsqu'ils se retrouvaient dans des contextes dans lesquels ils se sentaient compétents et où ils ressentaient une réciprocité sociale. Ainsi le développement de l'autodétermination des adolescents ayant un TSA les aide à s'affirmer, à exprimer leurs intérêts ou préférences face à leur avenir et aux moyens à mettre en place pour atteindre leurs objectifs. En conséquence, avec l'aide de leur famille et des intervenants scolaires ou sociaux, ils seront mieux outillés pour construire un avenir qui corresponde à leurs aspirations et surmonter les situations d'adversité qui peuvent se présenter.

Soutenir les familles pour faciliter la participation aux loisirs. Plusieurs familles ont de la difficulté à identifier des ressources ou à trouver de l'information quant à l'intégration communautaire de leur enfant ayant un TSA (Carter et al., 2013; Test et al., 2014) et se sentent démunies face au défi de cette intégration (Crabtree, 2007; Penney, 2013). Pour faciliter la participation sociale des jeunes ayant un TSA et outiller les familles quant aux possibilités d'activités communautaires et de sources de financement, Carter et al. (2013) évoquent la nécessité que les intervenants puissent soutenir les familles dans l'intégration communautaire afin de créer des liens entre le jeune et sa communauté. L'étude de Tétreault et al. (2012), réalisée auprès de familles québécoises, insiste sur le 
caractère prioritaire du soutien offert aux parents pour faciliter la participation aux loisirs des adolescents ayant des incapacités. Par exemple, il est recommandé de développer des ententes de collaboration entre les divers partenaires (municipal, communautaire ou scolaire) afin d'informer les parents quant au soutien financier pour le transport, de coordonner la mise en place de structures d'accompagnement pour les adolescents leur permettant de réaliser des activités dans un cadre adapté, etc. Les loisirs représentent une façon positive pour ces jeunes de contribuer à leur collectivité, et la participation à de telles activités leur permet de se connaitre, de s'épanouir et de s'engager dans de nouvelles activités (Tétreault et al., 2012).

Sensibiliser les pairs et leur donner des opportunités de socialiser avec les élèves qui ont un TSA. Les pairs gravitant autour de l'élève ayant un TSA peuvent être mal à l'aise d'interagir avec lui en raison de leur méconnaissance et de leur incompréhension des déficits sociaux associés au TSA (Bottema-Beutel et $\mathrm{Li}, 2015$; Jones et Frederickson, 2010). Ainsi, l'étude de Bottema-Beutel et Li (2015), réalisée à partir d'entrevues avec 38 pairs au développement typique (1318 ans) d'élèves ayant un TSA, suggère de mettre en place des stratégies de formation des pairs au sujet du TSA afin qu'ils soient en mesure de comprendre le comportement de ces élèves et d'apprendre comment interagir avec eux. Pour améliorer les relations sociales des jeunes ayant un TSA, il s'avère important, selon Shattuck et al. (2011), de favoriser leur participation dans des activités de groupe avec leurs pairs. Appuyant ces résultats, l'étude de Jones et Frederickson (2010), qui a examiné les caractéristiques comportementales d'une intégration réussie dans les classes ordinaires, fait ressortir l'importance de la mise en place d'un programme de sensibilisation. L'apparent manque d'habiletés sociales chez l'élève ayant un TSA pourrait ainsi ne pas être interprété de façon négative et les élèves au développement typique pourront être plus empathiques et témoigner davantage de considération à l'égard de ce pair. La présence fréquente des adolescents avec ou sans incapacités dans les milieux scolaires et communautaires permet de multiplier les occasions d'apprendre ensemble, de socialiser et de tisser des liens. L'adolescence est une période propice pour prendre une distance par rapport à la cellule familiale et développer des liens avec ses pairs, ce qui facilite le développement de l'identité et des habiletés pour vivre de façon autonome en société. En outre, plus les adolescents au développement typique ont d'occasions de mieux comprendre et d'interagir avec leurs camarades ayant un TSA, plus ils deviennent à l'aise d'échanger et de socialiser avec eux.

Sensibiliser la collectivité. Les résultats de l'étude de Crabtree (2007) font ressortir l'importance de la sensibilisation de la collectivité aux différences des enfants ayant des handicaps développementaux ou intellectuels. Les perceptions des participantes, exclusivement des mères, ont été explorées quant à l'acceptation de l'enfant par l'entourage et la collectivité. Il en ressort une grande préoccupation relativement à la sensibilisation du public aux différences et aux handicaps afin de réduire la ségrégation et d'augmenter l'acceptation des jeunes. Les caractéristiques des adolescents et jeunes adultes ayant un trouble du spectre de l'autisme sont très diversifiées et encore méconnues de l'ensemble de la population, ce qui laisse place à de fausses croyances et à des comportements de crainte ou d'évitement. C'est pourquoi la sensibilisation de la population et l'augmentation des contacts 
avec les personnes ayant un TSA est un élément incontournable de la réussite de leur insertion sociale et communautaire.

Offrir de la formation au sujet du TSA aux intervenants scolaires. La connaissance des caractéristiques du TSA par les intervenants scolaires est primordiale pour la réussite éducative de l'élève et facilite sa participation au sein de la classe (Brock et al., 2014; Penney, 2013). À cet égard, les résultats de l'étude de Brock et al. (2014), réalisée à partir de questionnaires remplis par divers intervenants scolaires de la maternelle au secondaire ( $\mathrm{N}: 456)$ aux ÉtatsUnis, ont permis d'éclairer les besoins du personnel scolaire en ce qui a trait à la connaissance et à la compréhension du TSA. Les enseignants, qui avaient en moyenne trois élèves ayant un TSA dans leur classe, n'étaient pas très confiants quant à leur capacité à mettre en œuvre des pratiques fondées sur des données probantes concernant ces élèves. Ainsi, la méconnaissance des interventions à mettre en place pour les soutenir par les enseignants et les éducateurs spécialisés représente un facteur de risque environnemental limitant la participation sociale de ces jeunes (Brock et al., 2014; Penney, 2013; Test et al., 2014). À cet effet, le besoin de formation continue est criant en raison de l'augmentation des élèves ayant un TSA inclus dans les classes ordinaires (Noiseux, 2008). Fait d'importance, le sujet le plus difficile à aborder par les enseignants était le développement vocationnel (Brock et al., 2014). Cet aspect revêt une importance capitale pour la participation sociale des élèves ayant un TSA, en favorisant l'accès à une vie active après la scolarisation (Boutin, 2012). II s'avère donc pertinent d'offrir de la formation aux intervenants scolaires afin de les outiller pour mieux soutenir ces élèves lors de la transition vers la vie adulte. Ils pourront leur offrir une éducation qui s'appuie sur des données probantes pour leur faciliter l'accès à des études postsecondaires ou les guider dans les activités du Parcours de formation axé sur l'emploi mis en place dans les écoles québécoises pour développer leurs habiletés socioprofessionnelles (Gouvernement du Québec, 2008).

Soutenir l'engagement de l'élève dans la planification de la transition de l'école à la vie active. Plusieurs études mettent en lumière l'importance de la planification de la transition de l'école vers la vie active (Carter et al., 2013; Chiang et al., 2013; Hagner et al., 2012; Hart et al., 2010; Hendricks et Wehman, 2009; Schall et al., 2006). Selon Hart et al. (2010), le plan de transition doit être centré sur la personne selon ses capacités, ses désirs et son projet de vie. Dans leur recension des écrits, Carter et al. (2013) recommandent la planification de la transition selon les intérêts, les compétences et les besoins de chaque jeune. Cette planification devrait être amorcée dès le début de la scolarisation au secondaire, vers l'âge de 14-15 ans (Hendricks et Wehman, 2009). II est aussi important que le jeune soit impliqué dans les décisions qui le concernent afin d'améliorer sa motivation. Le plan de transition sera également plus efficace s'il comprend des objectifs pour le jeune qui soient applicables dans sa communauté, donc en contexte naturel (Carter et al., 2013). Par exemple, le jeune pourrait s'inscrire à des activités sportives ou récréatives dans un centre communautaire, faire du bénévolat, poser sa candidature pour un emploi pendant la période estivale, etc. II faut donc que les intervenants scolaires ou sociaux gravitant autour de l'élève l'impliquent et soutiennent son engagement dans la planification de sa transition. Schall et al. (2006) ajoutent que les écoles accueillant des élèves ayant un TSA doivent inclure 
une gamme complète de services de transition, y compris des apprentissages concernant les activités de la vie quotidienne, la gestion des émotions, l'hygiène et les compétences académiques fonctionnelles. La participation de l'élève ayant un TSA à la planification de la transition doit ainsi comprendre l'accès aux services lui permettant d'acquérir les habiletés nécessaires à la réalisation de ses projets.

Permettre à l'élève d'explorer des possibilités d'emploi, des activités communautaires ou des options d'études postsecondaires. Pour que l'élève ayant un TSA puisse se positionner quant à son projet de vie et faire des choix, l'exploration des possibilités d'emploi (Chiang et al., 2013; Hagner et al., 2012; Schall et al., 2006; Test et al., 2014), des activités communautaires accessibles dans sa collectivité (Carter et al., 2013) et des options de formations postsecondaires (Carter et al., 2013; Hart et al., 2010; Taylor et Seltzer, 2011) est essentielle afin de lui permettre de connaitre ses préférences et confirmer ses choix. Puisqu'un des mandats des services éducatifs est de favoriser la participation sociale, l'exploration devrait inclure la visite des milieux que le jeune est susceptible de fréquenter à la fin de sa scolarisation. Le Parcours de formation axée sur l'emploi (Gouvernement du Québec, 2008) comprend la possibilité pour les élèves d'explorer et d'expérimenter diverses possibilités d'emploi, ce qui répond au besoin des élèves ayant un TSA de se projeter concrètement dans la réalité des milieux de travail. L'intégration réussie, significative et durable au sein d'un milieu de travail stimulant permet au jeune ayant un TSA de développer son estime de soi et son identité, en plus d'acquérir de nouvelles compétences (Hillier et al., 2007). Cette immersion lui permet d'avoir une vision réaliste des milieux de travail et de confirmer ses préférences dans la démarche de développement vocationnel. II est également possible que le jeune ayant un TSA désire poursuivre des études postsecondaires. À cet effet, les intervenants qui informent le jeune quant aux options possibles de formation postsecondaire lui permettent de faire un choix éclairé en ce qui concerne son avenir (Hart, Grigal et Weir, 2010). Être « étudiant » représente un rôle socialement valorisé en plus d'offrir l'occasion aux élèves ayant un TSA de fréquenter un établissement d'enseignement postsecondaire et de vivre une expérience sociale positive avec des pairs de leur âge (Hart et al., 2010). Chiang et al. (2013) mentionnent d'ailleurs qu'une meilleure participation sociale lorsque l'élève fréquente le milieu scolaire, à l'école et dans sa communauté, favoriserait une plus grande participation sociale lors du passage à la vie active.

Travailler ensemble (concertation, collaboration et partenariat). Plusieurs auteurs mettent en évidence l'importance des pratiques collaboratives ${ }^{6}$ entre la famille, le milieu scolaire et les services de santé et sociaux pour la participation sociale des jeunes ayant un TSA (Crabtree, 2007; Hendricks et Wehman, 2009; Penney, 2013; Schall et al., 2006; Tétreault, Beaupré, Carrière,

6. Les pratiques collaboratives réfèrent à un niveau élevé d'interdépendance professionnelle qui implique les membres d'une équipe qui travaillent ensemble, partagent leurs connaissances, leur expertise, leurs expériences, leurs habiletés et leurs compétences individuelles dans le but de résoudre des problèmes communs. II s'agit d'un type d'interaction impliquant au moins deux groupes de professionnels autour d'un but commun, dans un processus de communication visant la prise de décision et divers apprentissages (Beaumont, Lavoie et Couture, 2011). 
Freeman et Gascon, 2010). Les pratiques collaboratives permettent d'assurer un suivi dans les interventions auprès du jeune et de sa famille. Ce suivi est particulièrement important lors de la transition de l'école à la vie active, qui est souvent un moment où survient une rupture de services. L'ampleur des défis relatifs à la collaboration est soulignée dans l'étude québécoise de Tétreault et al. (2010) et des solutions sont apportées, notamment l'augmentation de la présence des intervenants de la santé et des services sociaux à l'école, car il s'agit du principal milieu de vie des élèves, après leur famille. De plus, les intervenants de services de réadaptation connaissent bien les élèves et leur famille, et ils possèdent des informations précieuses pour les accompagner dans leur trajectoire. Quant aux intervenants scolaires, le fait qu'ils côtoient les élèves six heures par jour en fait des partenaires privilégiés de leur formation scolaire et professionnelle. À cet effet, la production d'un plan de services individualisé et intersectoriel (PSII) pourrait permettre une coordination des services entre les diverses instances. Les adultes qui gravitent autour de l'adolescent ou du jeune adulte ayant un TSA l'ont soutenu et encouragé tout au long de son parcours scolaire, ils doivent se concerter en unissant leurs forces pour lui permettre d'actualiser son potentiel et de réaliser ses aspirations. Cette collaboration doit s'élargir au-delà des milieux scolaires et sociaux, en établissant des partenariats avec les organismes gouvernementaux et communautaires qui assurent un suivi auprès du jeune adulte et de sa famille (Test et al., 2014).

\section{Forces et limites de la recherche}

Considérant la présence de plus en plus grande des élèves ayant un TSA dans les classes régulières, cette recension des écrits a permis de mettre en lumière des stratégies éducatives pour favoriser leur participation sociale et par voie de conséquence, contribuer à la réussite de la transition de l'école à la vie active. Peu d'études en contexte québécois ont investigué cet enjeu, bien que son importance soit reconnue par les milieux scolaires et de réadaptation (Gouvernement du Québec, 2008; 2016; 2017), ce qui représente un aspect novateur de la présente recension des écrits. De plus, cette étude permet d'outiller les intervenants scolaires et sociaux en leur proposant des stratégies d'intervention éducatives concrètes et facilement applicables dans leur milieu d'intervention. Toutefois, puisque la participation sociale des adolescents et des jeunes adultes ayant un TSA est une préoccupation émergente et que peu de recherches ont été réalisées en contexte québécois ou canadien, certains facteurs de risque ou de protection, de même que les stratégies d'intervention recensées, gagneraient donc à être réexaminés en tenant compte du contexte culturel québécois.

\section{Conclusion}

Cette recension des écrits scientifiques a permis de mieux connaître les enjeux et défis de la participation sociale des adolescents et jeunes adultes ayant un TSA afin de mieux la soutenir et ainsi contribuer à une participation sociale optimale à la fin de la scolarisation. Les pratiques éducatives recensées comprennent des actions à privilégier dans une perspective systémique : avec ces jeunes, leur famille, les intervenants scolaires et les membres de leur collectivité. Elles soulignent l'importance du développement de la communication et des 
habiletés sociales de ces jeunes, à cette période critique de la transition vers la vie adulte. Plusieurs activités sont suggérées pour développer les habiletés sociales, basées sur des apprentissages qui sont réalisés de façon individuelle, dans le milieu familial, le milieu scolaire ou communautaire (avec des pairs ou les membres de leur collectivité). La nécessité de sensibiliser les pairs et la société en général est un élément incontournable de leur participation sociale et du développement de liens sociaux. Le milieu scolaire exerce un rôle prépondérant dans l'apprentissage à la vie adulte et au-delà de la sensibilisation, les intervenants scolaires ont besoin de formation afin d'offrir à ces jeunes une éducation de qualité qui leur permette de réaliser leurs aspirations. Finalement, les résultats confirment la nécessité d'une approche individualisée, basée sur leurs forces et leurs intérêts et un accompagnement qui soutienne leur engagement dans le processus de transition vers la vie adulte. Bien que les caractéristiques du TSA puissent représenter des entraves à leur participation sociale, ces jeunes ont des talents et des aspirations et peuvent se mobiliser lorsque l'environnement leur propose des conditions optimales d'apprentissage. Dans cette période critique de transition vers la vie adulte, les habiletés de communication et les habiletés sociales semblent être les compétences les plus étroitement liées à la participation sociale. Pour les jeunes ayant un TSA, la participation sociale est à la fois une forme d'apprentissage, de développement personnel et de contribution sociale, qui confirme leur appartenance à la collectivité et s'actualise en l'exerçant.

\section{Références}

American Psychiatric Association. (2015). DSM-5: manuel diagnostique et statistique des troubles mentaux (traduction M.-A. Crocq et J. D. Guelfi, 5 e éd.). Paris : Elsevier Masson.

Anderson, K. A., Shattuck, P. T., Cooper, B. P., Roux, A. M. et Wagner, M. (2014). Prevalence and correlates of postsecondary residential status among young adults with an autism spectrum disorder. Autism, 18(5), 562-570.

Beaumont, C., Lavoie, J. et Couture, C. (2011). Les pratiques collaboratives en milieu scolaire : cadre de référence pour soutenir la formation. Québec, QC : Centre de recherche et d'intervention sur la réussite scolaire (CRIRES).

Bottema-Beutel, K. et Li, Z. (2015). Adolescent judgments and reasoning about the failure to include peers with social disabilities. Journal of Autism and Developmental Disorders, 45(6), 18731886.

Brock, M. E., Huber, H. B., Carter, E. W., Juarez, A. P. et Warren, Z. E. (2014). Statewide assessment of professional development needs related to educating students with autism spectrum disorder. Focus on Autism and Other Developmental Disabilities, 29(2), 6779.

Broderick, C., Caswell, R., Gregory, S., Marzolini, S. et Wilson, O. (2002). 'Can I join the club?' A social integration scheme for adolescents with Asperger syndrome. Autism, 6(4), 427-431.

Brown, S.M. et Bebko, J.M. (2012). Generalization, overselectivity, and discrimination in the autism phenotype: A review. Research in Autism Spectrum Disorders, 6, 733-740.

Bruininks, R. H., Woodcock, R. W., Weatherman, R. F. et Hill, B. K. (1996). Scales of independent behavior revised. Rolling Meadows, IL: Riverside Publishing.

Carter, E. W., Harvey, M. N., Taylor, J. L. et Gotham, K. (2013). Connecting Youth and Young Adults with Autism Spectrum Disorders to Community Life. Psychology in the Schools, 50(9), 888898. 
Centers for Diseases Control and Prevention. (2015). Autism Spectrum Disors (ASD) - Data and Statistics. Washington, DC: U.S. Department of Health and Human Services.

Chen, Y.-W., Bundy, A. C., Cordier, R., Chien, Y.-L. et Einfeld, S. L. (2015). Motivation for everyday social participation in cognitively able individuals with autism spectrum disorder. Neuropsychiatric Disease and Treatment, 11, 2699-2709.

Chiang, H.-M., Cheung, Y. K., Li, H. et Tsai, L. Y. (2013). Factors associated with participation in employment for high school leavers with autism. Journal of Autism and Developmental Disorders, 43(8), 1832-1842.

Crabtree, S. A. (2007). Family responses to the social inclusion of children with developmental disabilities in the United Arab Emirates. Disability et Society, 22(1), 49-62.

Eaves, L. C. et Ho, H. H. (2008). Young adult outcome of autism spectrum disorders. Journal of Autism and Developmental Disorders, 38(4), 739-747.

Farley, M. A., McMahon, W. M., Fombonne, E., Jenson, W. R., Miller, J., Gardner, M., et al. (2009). Twenty-year outcome for individuals with autism and average or near-average cognitive abilities. Autism Research, 2(2), 109-118.

Fédération québécoise des centres de réadaptation en déficience intellectuelle et en troubles envahissant du développement. (2013). La participation sociale des personnes présentant une déficience intellectuelle ou un trouble envahissant du développement: $d u$ discours à une action concertée. Montréal, QC : FQCRDITED.

Fougeyrollas, P. (2010). Le funambule, le fil et la toile: transformations réciproques $d u$ sens $d u$ handicap. Québec, QC : Presses de I'Université Laval.

Freeth, M., Bullock, T. et Milne, E. (2013). The distribution of and relationship between autistic traits and social anxiety in a UK student population. Autism, 17(5), 571581.
Glutting, J. J., Adams, W. et Sheslow, D. (2000). Wide Range Intelligence Test. Wilmington, DE: Wide Range.

Goldingay, S., Stagnitti, K., Sheppard, L., McGillivray, J., McLean, B. et Pepin, G. (2015). An intervention to improve social participation for adolescents with autism spectrum disorder: Pilot study. Developmental Neurorehabilitation, 18(2), 122-130.

Gouvernement du Québec. (2017). Vers une meilleure intégration des soins et des services pour les personnes ayant une déficience. Cadre de référence pour l'organisation des services en déficience physique, déficience intellectuelle et trouble du spectre de l'autisme. Québec, QC : Ministère de la Santé et des Services sociaux.

Gouvernement du Québec. (2016). Bilan des orientations ministérielles en déficience intellectuelle et actions structurantes pour le programme-services en déficience intellectuelle et trouble $d u$ spectre de l'autisme. Québec, QC : Ministère de la Santé et des Services sociaux.

Gouvernement du Québec. (2008). Parcours de formation axée sur l'emploi. Québec, QC : Ministère de l'Éducation, du Loisir et du Sport.

Gouvernement du Québec. (2006). Programme de formation de l'école québécoise. Québec, QC : Ministère de l'Éducation.

Gray, K. M., Keating, C. M., Taffe, J. R., Brereton, A. V., Einfeld, S. L., Reardon, T. C. et Tonge, B. J. (2014). Adult outcomes in autism: Community inclusion and living skills. Journal of Autism and Developmental Disorders, 44(12), 3006-3015.

Hagner, D., Kurtz, A., Cloutier, H., Arakelian, C., Brucker, D. L. et May, J. (2012). Outcomes of a family-centered transition process for students with autism spectrum disorders. Focus on Autism and Other Developmental Disabilities, 27(1), 42-50. 
Hall, S. A. (2009). The social inclusion of people with disabilities: A qualitative meta-analysis. Journal of Ethnographic \& Qualitative Research, 3, 162-173.

Hall, S. A. (2010). The social inclusion of young adults with intellectual disabilities: A phenomenology of their experiences. Thèse de doctorat, University of Nebraska, Lincoln.

Hart, D., Grigal, M. et Weir, C. (2010). Expanding the paradigm: Postsecondary education options for individuals with autism spectrum disorder and intellectual disabilities. Focus on Autism and Other Developmental Disabilities, 25(3), 134-150.

Hendricks, D. R. et Wehman, P. (2009). Transition from school to adulthood for youth with autism spectrum disorders: Review and recommendations. Focus on Autism and Other Developmental Disabilities, 24(2), 77-88.

Hetherington, S. A., Durant-Jones, L., Johnson, K., Nolan, K., Smith, E., Taylor-Brown, S. et Tuttle, J. (2010). The lived experiences of adolescents with disabilities and their parents in transition planning. Focus on Autism and Other Developmental Disabilities, 25(3), 163-172.

Hillier, A., Campbell, H., Mastriani, K., Izzo, M. V., Kool-Tucker, A. K., Cherry, L. et Beversdorf, D. O. (2007). Two-year evaluation of a vocational support program for adults on the autism spectrum. Career Development for Exceptional Individuals, 30(1), 35-47.

Huws, J. C. et Jones, R. S. P. (2015). 'I'm really glad this is developmental': Autism and social comparisonsAn interpretative phenomenological analysis. Autism, 19(1), 84-90.

Ionescu, S. (2015). De la résilience naturelle à la résilience assistée. Dans $F$. Julien-Gauthier et C. Jourdan-Ionescu (dir.), Résilience assistée, réussite éducative et réadaptation (p. 5-18). Québec: Livres en ligne du CRIRES. En ligne http://lel.crires.ulaval.ca/public/ resilience.pdf.
Ionescu, S. (2016). Résiliences: ressemblances dans la diversité. Paris : Odile Jacob.

Ionescu, S. (2011). Traité de résilience assistée. Paris : Presses Universitaires de France.

Jones, A. P. et Frederickson, N. (2010). Multiinformant predictors of social inclusion for students with Autism spectrum disorders attending mainstream school. Journal of Autism and Developmental Disorders, 40(9), 1094-1103.

Jourdan-Ionescu, C. (2001). Intervention écosystémique individualisée axée sur la résilience. Revue québécoise de psychologie, 22(1), 163-186.

Jourdan-Ionescu, C. (2017). La résilience assistée. Formation offerte à l'Université Catholique d'Afrique Centrale (UCAC). Yaoundé, Cameroun.

Jourdan-Ionescu, C. et Julien-Gauthier, F. (2011). Clés de résilience en déficience intellectuelle. Dans S. Ionescu (Éd.), Traité de résilience assistée (p. 283325). Paris: Presses Universitaires de France.

Julien-Gauthier, F., Jourdan-lonescu, C., Martin-Roy, S., Ruel, J. et Legendre, M. P. (2014). The assessment of the resilience of people with cognitive impairments or intellectual disabilities. From Person to Society, 1079.

Julien-Gauthier, F., Héroux, J. et JourdanIonescu, C. (2012). L'insertion sociale et professionnelle des jeunes adultes qui ont des incapacités. Développement humain, handicap et changement social, 20(1), 91-99.

Julien-Gauthier, F., Jourdan-lonescu, C., Ruel, J., Martin-Roy, S. et Legendre, M.P. (2012). L'évaluation de la résilience des personnes ayant une déficience intellectuelle. Paper presented at the Xlle Congrès de l'association Internationale de recherche scientifique en faveur des personnes handicapées mentales, Mont-Tremblant, QC.

Julien-Gauthier, F., Ruel, J., Moreau, A. et Martin-Roy, S. (2016). La transition de l'école à la vie adulte d'une élève ayant 
une déficience intellectuelle légère. Enfance en difficulté, 4, 53-101.

Julien-Gauthier, F., Martin-Roy, S., Moreau, A., Ruel, J., et Rouillard-Rivard, D. (2016). La participation sociale de jeunes adultes ayant des incapacités intellectuelles, un an après la fin de la scolarisation. Revue internationale de communication et socialisation, 3(2), 155-180.

Kreider, C. M., Bendixen, R. M., Mann, W. C., Young, M. E. et McCarty, C. (2015). Mixed-method exploration of social network links to participation. OTJR: Occupation, Participation and Health, 35(3), 151-159.

Kuo, M. H., Orsmond, G. I., Cohn, E. S. et Coster, W. J. (2013). Friendship characteristics and activity patterns of adolescents with an autism spectrum disorder. Autism, 17(4), 481-500.

Liebowitz, M. R. (1987). Social phobia. Modern Problems of Pharmacopsychiatry, 22, 141-173.

Liptak, G. S., Kennedy, J. A. et Dosa, N. P. (2011). Social participation in a nationally representative sample of older youth and young adults with autism. Journal of Developmental and Behavioral Pediatrics, 32(4), 277-283.

Lysaght, R., Cobigo, V. et Hamilton, K. (2012). Inclusion as a focus of employment-related research in intellectual disability from 2000 to 2010: A scoping review. Disability et Rehabilitation, 34(16), 1339-1350. doi: 10.3109/09638288.2011.644023

Martin-Roy, S. et Julien-Gauthier, F. (2017). Accompagner les élèves ayant des incapacités intellectuelles lors de la transition de l'école à la vie active. Initio, Revue sur l'éducation et la vie au travail (6), 31-51.

Myers, E., Davis, B. E., Stobbe, G. et Bjornson, K. (2015). Community and social participation among individuals with autism spectrum disorder transitioning to adulthood. Journal of Autism and Developmental Disorders, 45(8), 2373-2381.
Nicholas, D. B., Attridge, M., Zwaigenbaum, L. et Clarke, M. (2015). Vocational support approaches in autism spectrum disorder: A synthesis review of the literature. Autism, 19(2), 235-245.

Noiseux, M. (2008). Surveillance des troubles envahissants du développement chez les enfants de 4 à 17 ans de la Montérégie, 2000-2001 à 2007-2008. Agence de la santé et des services sociaux de la Montérégie.

Novak, A.A., Stancliffe, R. J., McCarron, M. et McCallion, P. (2013). Social inclusion and community participation of individuals with intellectual/ developmental disabilities. Intellectual and Developmental Disabilities, 51(5), 360-375. doi: 10.1352/1934-955651.5 .360

Orsmond, G. I., Krauss, M. W. et Seltzer, M. M. (2004). Peer relationships and social and recreational activities among adolescents and adults with autism. Journal of Autism and Developmental Disorders, 34(3), 245-256.

Orsmond, G. I., Shattuck, P. T., Cooper, B. P., Sterzing, P. R. et Anderson, K. A. (2013). Social participation among young adults with an autism spectrum disorder. Journal of Autism and Developmental Disorders, 43(11), 27102719.

Penney, S. C. (2013). Qualitative investigation of school-related issues affecting individuals diagnosed with autism spectrum disorder and cooccurring anxiety and/or depression. Autism Insights, 5, 75-91.

Piers, E.V. (1984) Piers-Harris Children's Self Concept Scale: Revised Manual. Los Angeles, CA: Western Psychological Services.

Proulx, J. (2008). Qualité de vie et participation sociale: deux concepts clés dans le domaine de la déficience intellectuelle - Une recension des écrits. Dans Laboratoire de recherche sur les pratiques et les politiques sociales (dir.) : Université du Québec à Montréal, Montréal, QC. 
Rouillard-Rivard, D. (2016). Stratégies d'intervention pour favoriser la participation sociale des adolescents et jeunes adultes (14 à 21 ans) ayant un trouble du spectre de l'autisme. (Essai de maitrise en psychoéducation), Université Laval, Québec, QC.

Rouillard-Rivard, D., Julien-Gauthier, F. et Martin-Roy, S. (août 2016). Stratégies d'intervention pour favoriser la participation sociale des adolescents et jeunes adultes ayant un trouble du spectre de l'autisme. Communication présentée dans le cadre du 3ième Congrès mondial sur la résilience. Trois-Rivières.

Schall, C., Cortijo-Doval, E., Sherron Targett, P. et Wehman, P. (2006). Applications for Youth with Autism Spectrum Disorders. Dans P. Wehman (dir.), Life beyond the classroom: transition strategies for young people with disabilities (4th ed., pp. 535-575). Baltimore: Paul H. Brookes.

Seltzer, M. M. et Krauss, M. W. (1989). Aging parents with adult mentally retarded children: family risk factors and sources of support. American Journal on Mental Retardation, 94, 303-312.

Shattuck, P. T., Orsmond, G. I., Wagner, M. et Cooper, B. P. (2011). Participation in social activities among adolescents with an autism spectrum disorder. PLOS ONE, 6(11).

St-Georges, J. (2017). La participation sociale de jeunes adultes ayant une déficience intellectuelle (18-21 ans) lors de la transition de l'école à la vie adulte. (Essai de maitrise en psychoéducation), Université Laval, Québec, QC.

Taylor, J. L. et Seltzer, M. M. (2011). Employment and post-secondary educational activities for young adults with autism spectrum disorders during the transition to adulthood. Journal of Autism and Developmental Disorders, 41(5), 566-574.

Test, D. W., Smith, L. E. et Carter, E. W. (2014). Equipping youth with autism spectrum disorders for adulthood: Promoting rigor, relevance, and relationships. Remedial and Special Education, 35(2), 80-90.

Tétreault, S., Beaupré, P., Carrière, M., Freeman, A. et Gascon, H. (2010). L'évaluation de l'implantation et des effets de l'Entente de complémentarité entre le réseau de la santé et des services sociaux et le réseau de l'éducation. Rapport scientifique intégral. Québec, QC : Université Laval.

Tétreault, S. (2014). Recension des écrits. Dans S. Tétreault et P. Guillez (Eds.), Guide pratique de recherche en réadaptation (pp. 137-149). Louvain-laNeuve, Belgique : De Boeck.

Tétreault, S. (2015). Défis et enjeux de la participation sociale. Communicaiton présentée à la journée d'échange : Transition de l'école à la vie active pour les jeunes ayant des incapacités. http://www.irdpq.qc.ca/sites/default/ files/images/programme_journee_13 avril_2015_final.pdf

Tétreault, S., Beaupré, P., Boucher, N., Carrière, M., Gascon, H., Piérart, G. et Marier Deschênes, P. (2012). Recommandations de stratégies de soutien : pour assurer l'épanouissement des familles québécoises vivant avec un enfant handicapé (de la naissance à 17 ans). Québec : Office des personnes handicapées du Québec (OPHQ).

Tobin, M. C., Drager, K. D. R. et Richardson, L. F. (2014). A systematic review of social participation for adults with autism spectrum disorders: Support, social functioning, and quality of life. Research in Autism Spectrum Disorders, 8(3), 214-229.

Wang, H.-Y. et Berg, C. (2014). Participation of young adults with high-functioning autism in Taiwan: a pilot study. OTJR: Occupation, Participation and Health, 34(1), 41-51.

Wehman, P., Schall, C., Carr, S., Targett, P., West, M. et Cifu, G. (2014). Transition from school to adulthood for youth with autism spectrum disorder: What we know and what we need to know. Journal of Disability Policy Studies, 25(1), 30-40. 